\title{
Photosynthetically Controlled Spirulina, but Not Solar Spirulina, Inhibits TNF-a Secretion: Potential Implications for COVID-19-Related Cytokine Storm Therapy
}

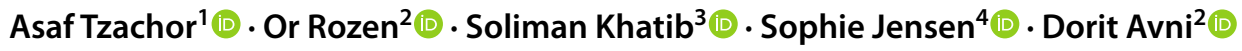

Received: 12 January 2021 / Accepted: 13 January 2021 / Published online: 10 February 2021

(c) The Author(s) 2021

\begin{abstract}
An array of infections, including the novel coronavirus (SARS-CoV-2), trigger macrophage activation syndrome (MAS) and subsequently hypercytokinemia, commonly referred to as a cytokine storm (CS). It is postulated that CS is mainly responsible for critical COVID-19 cases, including acute respiratory distress syndrome (ARDS). Recognizing the therapeutic potential of Spirulina blue-green algae (Arthrospira platensis), in this in vitro stimulation study, LPS-activated macrophages and monocytes were treated with aqueous extracts of Spirulina, cultivated in either natural or controlled light conditions. We report that an extract of photosynthetically controlled Spirulina (LED Spirulina), at a concentration of $0.1 \mu \mathrm{g} / \mathrm{mL}$, decreases macrophage and monocyte-induced TNF- $\alpha$ secretion levels by over $70 \%$ and $40 \%$, respectively. We propose prompt in vivo studies in animal models and human subjects to determine the putative effectiveness of a natural, algae-based treatment for viral CS and ARDS, and explore the potential of a novel anti-TNF- $\alpha$ therapy.
\end{abstract}

Keywords COVID-19 $\cdot$ Cytokine storm $\cdot$ Immunology $\cdot$ Biotechnology $\cdot$ Spirulina $\cdot$ Anti TNF- $\alpha$ therapy

\section{Introduction}

The novel coronavirus disease (COVID-19) is an emerging contagious respiratory tract illness caused by the severe acute respiratory syndrome coronavirus 2 (SARS-CoV-2) (Hu et al. 2020). From late 2019, SARS-CoV-2 infections have resulted in an array of clinical responses which differ between individual cases, from asymptomatic conditions

Asaf Tzachor and Or Rozen contributed equally to this work.

Dorit Avni

dorita@migal.org.il

1 Centre for the Study of Existential Risk \& Cambridge Global Food Security Research Center, University of Cambridge, Cambridge, UK

2 Sphingolipids, Active Metabolites and Immune Modulation Laboratory, MIGAL - Galilee Research Institute, Kiryat Shemona, Israel

3 Natural compounds and analytical chemistry Laboratory, MIGAL - Galilee Research Institute and Tel Hai college, Kiryat Shemona, Israel

4 MATIS - Food and Biotech Research and Development, Reykjavík, Iceland to detrimental manifestations and mortality. Whereas precise immune-pathological processes that COVID-19 activates remain, as of yet, contested (Coperchini et al. 2020), there is an ostensible agreement on the major mechanism by which the virus causes severe symptoms.

Epidemiological studies have indicated that exposure to the etiologic agent SARS-CoV-2 provokes macrophages and monocytes to release an excessive amount of different pro-inflammatory cytokines, such as tumor necrosis factor (TNF)- $\alpha$ and interleukin (IL)-6, to cause a hypercytokinemia, commonly referred to as a cytokine storm (CS) (Ishikawa 2012; Ye et al. 2020).

An influx of TNF- $\alpha$, as part of the CS, destabilizes endothelial cell networks and induces damage of vascular barrier, capillary damage, diffuse alveolar damage (DAD), apoptotic cell death, and multi-organ failure. Furthermore, a recent analysis indicated higher systemic levels of IL-2, IL-7, IL-10, monocyte chemoattractant protein-1 (MCP1), macrophage inflammatory protein-1A (MIP-1A), and TNF- $\alpha$, among critically ill COVID-19 patients (McGonagle et al. 2020; Ruan et al. 2020).

Specifically, excess release of TNF- $\alpha$ plays a critical role in disrupting the lung endothelial and epithelial barriers, which may cause acute respiratory distress 
syndrome (ARDS) (Shimizu 2019). ARDS requires admission to intensive care units (ICU), where invasive mechanical ventilation may be administered (Mittermaier et al. 2020). Furthermore, it is generally accepted that ARDS is the main cause of death of patients with COVID (Mehta et al. 2020). The prevalence of COVID-19 ARDS incidences led to a global public health emergency, prompting governments to suspend social activities and impose unprecedented movement restriction and social distancing. These measures were set to maintain ICUs within their operational capacity limitations, a pending public healthcare concern (Alkuzweny et al. 2020; Moghadas et al. 2020).

Considering the role of TNF- $\alpha$ in triggering COVID-19related cytokine storm syndrome (COVID-CS) and ARDS, it is necessary to develop new approaches for anti-TNF therapy. Indeed, since the outbreak of the pandemic, TNF- $\alpha$ blockers have shown promising outcomes in treating, and mitigating, severe illness (Robinson et al. 2020).

Herein, a novel approach is proposed for TNF- $\alpha$ inhibition, based on a treatment with the blue-green algae Spirulina (Arthrospira platensis) extract.

The potential health benefits of Spirulina are well documented (Belay et al. 1993; Furmaniak et al. 2017). This blue-green algae contains C-phycocyanin (C-PC), a pigment-binding protein, which enhances antioxidation, anti-inflammation, and anti-tumor activities (Cian et al. 2012; Saini and Sanyal 2015). Furthermore, Spirulina may be cultivated in different conditions and extracted using various techniques, which may affect the bioactive metabolite content of Spirulina (Minhas et al. 2016). Under certain conditions, for instance, irradiation by light-emitting diodes (LED) to control photosynthesis, algal bioactivity such as anti-inflammatory properties may be enhanced (De Morais et al. 2015; Ooms et al. 2016).

In this study, we exposed macrophages and monocytes activated by the pathogenic stimulator lipopolysaccharide (LPS) to different doses of Spirulina extracts, cultivated in either full-range solar spectrum or controlled light conditions. We report that an aqueous extract of a photosynthetically controlled Spirulina (LED Spirulina) inhibits TNF- $\alpha$ secretion by over $70 \%$ from LPS-activated macrophages and over $40 \%$ from LPS-activated monocyte cells.

\section{Materials and Methods}

\section{Reagents}

Cell lines for measuring anti-inflammatory activity human monocyte (THP-1) and murine macrophages (RAW 264.7) were used. All were provided by the American Type Culture Collection (ATCC, USA). Additionally, Rosewell Park
Memorial Institute (RPMI) 1640, Dulbecco's Modified Eagle Medium (DMEM) were purchased from ATCC. Fetal bovine serum (FBS), penicillin-streptomycin, sodium pyruvate, and glutamine were purchased from Biological Industries (Beit HaEmek, Israel). 2-Mercaptoethanol was purchased from BIORAD and ELISA kits were purchased from PeproTech. LPS was purchased from Sigma-Aldrich (Sigma-Aldrich, Israel).

\section{Culture and Extraction}

Spirulina (UTEX 3086) was cultivated in Zarrouk medium (Rajasekaran et al. 2016). Cultivation was carried out in 180-L flat panel airlift photobioreactors. The cultures were maintained under agitation induced by insufflation of filtered air, with flow aeration of $0.5 \mathrm{vvm}$ (air volume/medium volume/minute).

Temperature was kept at $31 \pm 2{ }^{\circ} \mathrm{C}$ and $\mathrm{pH}$ was maintained at $10.8 \pm 0.2^{\circ} \mathrm{C}$. Cultures were grown under two illumination conditions: (A) full-range solar spectrum at irradiance of $750 \mu \mathrm{mol} /\left(\mathrm{m}^{2} \mathrm{~s}\right)$, here referred to as "Solar Spirulina", and (B) red/blue/UV at irradiance of $750 \mu \mathrm{mol} /\left(\mathrm{m}^{2} \mathrm{~s}\right.$ ) (USP \# 63/026,764), here referred to as "LED Spirulina".

The irradiance was measured on the surface of the flask using a LI-250A light meter (Nebraska, USA) and a Li-Cor quantum sensor. Both cultures were water-extracted using physical freeze-thawing for cellular disruption (Chu et al. 2010), obtaining one Solar Spirulina extract and one LED Spirulina extract.

\section{Metabolomics Profile of the Full Spectrum and LED Spirulina Extracts}

Solar and LED Spirulina extracts were dissolved in methanol $(100 \mu \mathrm{g} / \mathrm{mL})$ and $5 \mu \mathrm{L}$ of the solutions were injected into a UHPLC connected to a photodiode array detector (Dionex Ultimate 3000), with a reverse-phase column (ZORBAX Eclipse plus $\left.\mathrm{C} 18,3.0 \times 100^{\circ} \mathrm{mm}, 1.8^{\circ} \mu \mathrm{m}\right)$.

The mobile phase consisted of (A) DDW with $0.1 \%$ formic acid and (B) acetonitrile containing $0.1 \%$ formic acid. The gradient started with $2 \% \mathrm{~B}$ then increased to $30 \% \mathrm{~B}$ in $4 \mathrm{~min}$, then increased to $40 \% \mathrm{~B}$ in $1 \mathrm{~min}$ and kept isocratic at $40 \% \mathrm{~B}$ for $3 \mathrm{~min}$. Then increased to $98 \% \mathrm{~B}$ in $6 \mathrm{~min}$ and kept isocratic at $98 \% \mathrm{~B}$ for $9 \mathrm{~min}$.

Phase B returned to 5\% in 3 min and the column allowed to equilibrate at $5 \% \mathrm{~B}$ for $5 \mathrm{~min}$ before the next injection. The flow rate was $0.4 \mathrm{~mL} / \mathrm{min}$. MS/MS analysis performed with heated electrospray ionization (HESI-II) source connected to a Q Exactive ${ }^{\mathrm{TM}}$ Plus Hybrid Quadrupole-Orbitrap ${ }^{\mathrm{TM}}$ Mass Spectrometer Thermo Scientific ${ }^{\mathrm{TM}}$. ESI capillary voltage was set to $3500 \mathrm{~V}$, capillary temperature to $300{ }^{\circ} \mathrm{C}$, gas temperature to $350{ }^{\circ} \mathrm{C}$, and gas flow to $35 \mathrm{~mL} / \mathrm{min}$.

The mass spectra (m/z 67-1000) acquired in negative and positive-ion mode with high resolution $(\mathrm{FWHM}=70,000)$. 
Data-dependent MS/MS was performed with collision energy of 15,50 , and $100 \mathrm{eV}$.

\section{Measurement of the Secretions of Pro-inflammatory Cytokines from RAW264.7 Macrophages}

The amounts of TNF- $\alpha$ and IL- 6 secreted from mouse macrophage cells were measured with TNF- $\alpha$ and IL-6 ELISA kits (PeproTech) according to manufacturer instructions. First, RAW264.7 cells $\left(1 \times 10^{5}\right.$ cells/well $)$ were inoculated into a 96-well plate and cultured for $48 \mathrm{~h}$ in DMEM 5\% FBS $(v / v)$ and penicillin-streptomycin $(100 \mathrm{IU} / \mathrm{mL}$ and $1 \mu \mathrm{g} / \mathrm{mL})$ at $37{ }^{\circ} \mathrm{C}$ with $5 \% \mathrm{CO}_{2}$. Various concentrations of the Spirulina extracts were added 20 min prior to LPS $(100 \mathrm{ng} / \mathrm{mL})$ stimulation for additional $4 \mathrm{~h}$ of culturing. The concentrations of the cytokines were measured in the ELISA reader infinite M200 PRO (TECAN, Switzerland) at $450 \mathrm{~nm}$ with correction at $620 \mathrm{~nm}$.

\section{Measurement of the Secretions of Pro-inflammatory Cytokines from Human THP-1 Monocyte Cells}

The amount of TNF- $\alpha$ secreted from human monocyte cells was determined with TNF- $\alpha$ ELISA kit (PeproTech) according to manufacturer instructions. THP- 1 cells $\left(1 \times 10^{5}\right.$ cells/well) were inoculated into a 96-well plate and cultured for $48 \mathrm{~h}$ in RPMI 1640 culture medium supplemented with $10 \%(v / v)$ heat-inactivated FBS, $1 \%$ sodium pyruvate $(11.0 \mathrm{mg} / \mathrm{mL}(100 \mathrm{mM})), 0.05 \mu \mathrm{M}$ 2-mercaptoethanol, and $1 \%$ penicillin-streptomycin $(100 \mathrm{IU} / \mathrm{mL}$ and $1 \mu \mathrm{g} /$ $\mathrm{mL}$ ) at $37{ }^{\circ} \mathrm{C}$ with $5 \% \mathrm{CO}_{2}$. Various concentrations of Spirulina extracts were added $20 \mathrm{~min}$ prior to LPS (100 ng/ $\mathrm{mL})$ stimulation for additional $2 \mathrm{~h}$ of culturing. The concentrations of the cytokines were measured in the ELISA reader infinite M200 PRO (TECAN, Switzerland) at $450 \mathrm{~nm}$ with correction at $620 \mathrm{~nm}$.

\section{Data Preprocessing}

Peak annotation and peak area integration performed with Compound Discoverer 3.1 (Thermo Xcalibur, Version 3.1.0.305). Compound putative identification was performed based on MS spectral data using MzCloud database and HRMS and isotope profile data using ChemSpider database.

\section{Statistical Analysis}

All in vitro experimental data was obtained from three separate experiments performed in three replicates, and statistical significance was determined by one-way analysis of variance (ANOVA) and post hoc Tukey, using the Statistical Analysis System program, PRISMA 8. The difference between the significance levels was set to $p<0.05$.

\section{Results}

\section{Metabolomics Profile from Spirulina Extracts of Two Different Cultivation Conditions}

The metabolomics profile from Spirulina extracts was determined using un-targeted LC/MS/MS. PC analysis of the 109 detected features (metabolites) is displayed in Fig. 1a. The first two principal components of the PCA score plot were responsible for $62.1 \%$ (43.8\% for PC1 and $18.3 \%$ for PC2) of the overall variance of the metabolomics profile, showing a clear separation between the Solar and LED Spirulina extracts. Seven compounds were significantly upregulated in the LED extract compared with Solar extract while 23 compounds downregulated (Fig. 1b). Two of the upregulated compounds, sorbitol and adenosine derivate, annotated based on MS/MS spectra using mzCloud database with MS/MS spectral similarity of $97 \%$ and $91 \%$, respectively. These bioactive compounds significantly increased in the LED extract by 1.7 - and 4.8 -fold with $P$ value of 0.01 and $7.8 \times 10^{-10}$, respectively.

In addition, $\mathrm{C}$-phycocyanin (CPC) bioactive compound was also significantly increased in the LED extract, by 4.7 -fold.

\section{Inhibition of Pro-inflammatory Cytokine Secretion by the Extracts}

Monocytes and macrophages play a pivotal role in COVIDrelated CS; therefore, the inhibitory effect of the extracts related to CS was tested in both macrophages and monocyte cells.

Our results illustrate the effects of the two extracts (Solar Spirulina and LED Spirulina) on LPS-induced secretion of TNF- $\alpha$ from mouse macrophages. LED Spirulina shows a $70 \%$ reduction in TNF- $\alpha$ levels at the concentration of $0.1 \mu \mathrm{g} / \mathrm{mL}$ (Fig. 2b), while no inhibitory effect was observed for Solar spirulina (Fig. 2a). In contrast to the inhibitory effect on TNF- $\alpha$, LED Spirulina had no effect on the secretion of IL-6 at $0.1 \mu \mathrm{g} / \mathrm{mL}$ (Fig. 2d). Moreover, results indicate that at higher dose of LED Spirulina extract $(10 \mu \mathrm{g} /$ $\mathrm{mL}$ ), there was a minor effect on IL-6 secretion (Fig. 2d), while no inhibitory effect was observed on TNF- $\alpha$ (Fig. 2b). Solar Spirulina had no effect on IL-6 secretion (Fig. 2c).

Since monocytes contribute to COVID-CS (Jafarzadeh et al. 2020), the effect of the different Spirulina extracts was also assessed in human monocyte-stimulated cells (Fig. 3a, b). The LED-spirulina extract inhibitory effect was also observed in LPS-stimulated human monocyte, reducing TNF- $\alpha$ levels over $40 \%$ at the indicated concentrations.

In addition, the extracts in the absence of LPS did not exhibit off target effect and TNF- $\alpha$ or IL- 6 was not detected in non-LPS-stimulated macrophages and monocyte cells. 




Fig. 1 Multivariate statistical analyses. The principal component analysis (PCA) score plots of the Solar and LED Spirulina extracts (a). Differential analysis that presents all the difference of the metabolite profile between Solar and LED Spirulina extracts (b). Dots in red are the upregulated compounds in the LED extract compared with

\section{Discussion}

As reported in previous studies (Feldmann et al. 2020), critically ill COVID-19 patients who were administered a single dose of a TNF- $\alpha$ neutralizing antibody were $45 \%$ less likely to die overall, and more likely to be weaning from mechanical ventilation 1 month after treatment, compared with untreated patients. This suggests that control of CS in its early stage through such means as immunomodulators and cytokine antagonists, as well as reduction of lung inflammatory cell infiltration, is key to reducing COVID-19-related mortality rates.

This study showed that LED Spirulina extract in low doses is able to decrease excessive release of TNF- $\alpha$ in LPSactivated macrophages and LPS-activated monocyte cells by over $70 \%$ and $40 \%$, respectively. If further clinical trials confirm these efficacy rates among human subjects, then LED Spirulina may act as a novel TNF- $\alpha$ suppressor.

The substantial inhibitory effect of LED Spirulina on TNF- $\alpha$ secretion, in contrast to an unsubstantial effect on IL-6 release, prompts us to assume that the LED Spirulina extract serves as a specific TNF- $\alpha$ suppressor.

It should be emphasized that LED Spirulina's anti-TNF- $\alpha$ effect exhibited a reversed dose-response activity. This might suggest that very low doses of LED Spirulina extract-related active molecules can affect endpoints (Vandenberg 2014). A similar effect was shown in other plant-based whole extracts,
Solar extract. Dots in green are the downregulated compounds in the LED extract compared with Solar extract. Squared metabolites are significantly increased (red) or decreased (green) at the LED extract compared with Solar extract with $P$ value $<0.05$ using $t$ test statistical analysis

exhibiting the highest activity at the lowest concentration (Otegbade et al. 2017).

A possible explanation for the reaction pattern observed in this study for TNF- $\alpha$ and IL-6 inhibition is that different bioactive molecules of LED Spirulina extract at different absolute quantities affect macrophages and monocyte cells differently, with some bioactive molecules suppressing TNF- $\alpha$ at low dosages, while others inhibit IL- 6 release only at higher dosages.

In addition, since both Solar and LED Spirulina extracts include CPC, the cytokine inhibitory bioactivity of the LED Spirulina extract could not be explained by the presence of this molecule alone (Jiang et al. 2017). Furthermore, according to previous studies (Cherng et al. 2007), using pure CPC on LPS-activated macrophages results in $40 \%$ TNF- $\alpha$ inhibition at $250 \mu \mathrm{g} / \mathrm{mL}$. Here, we show inhibitory effect of $70 \%$ at $0.1 \mu \mathrm{g} / \mathrm{mL}$ of LED extract, suggesting that $\mathrm{CPC}$ is not the only responsible compound for $\mathrm{TNF}-\alpha$ inhibition. As there are indications that sorbitol and adenosine derivate have anti-inflammatory properties (Mongkhon et al. 2014), the upregulation of these groups in LED Spirulina compared with Solar Spirulina leads us to suggest that the bioactivity of LED Spirulina extract results from a synergistic effect between several functional groups that might include CPC, sorbitol, and adenosine derivate. 

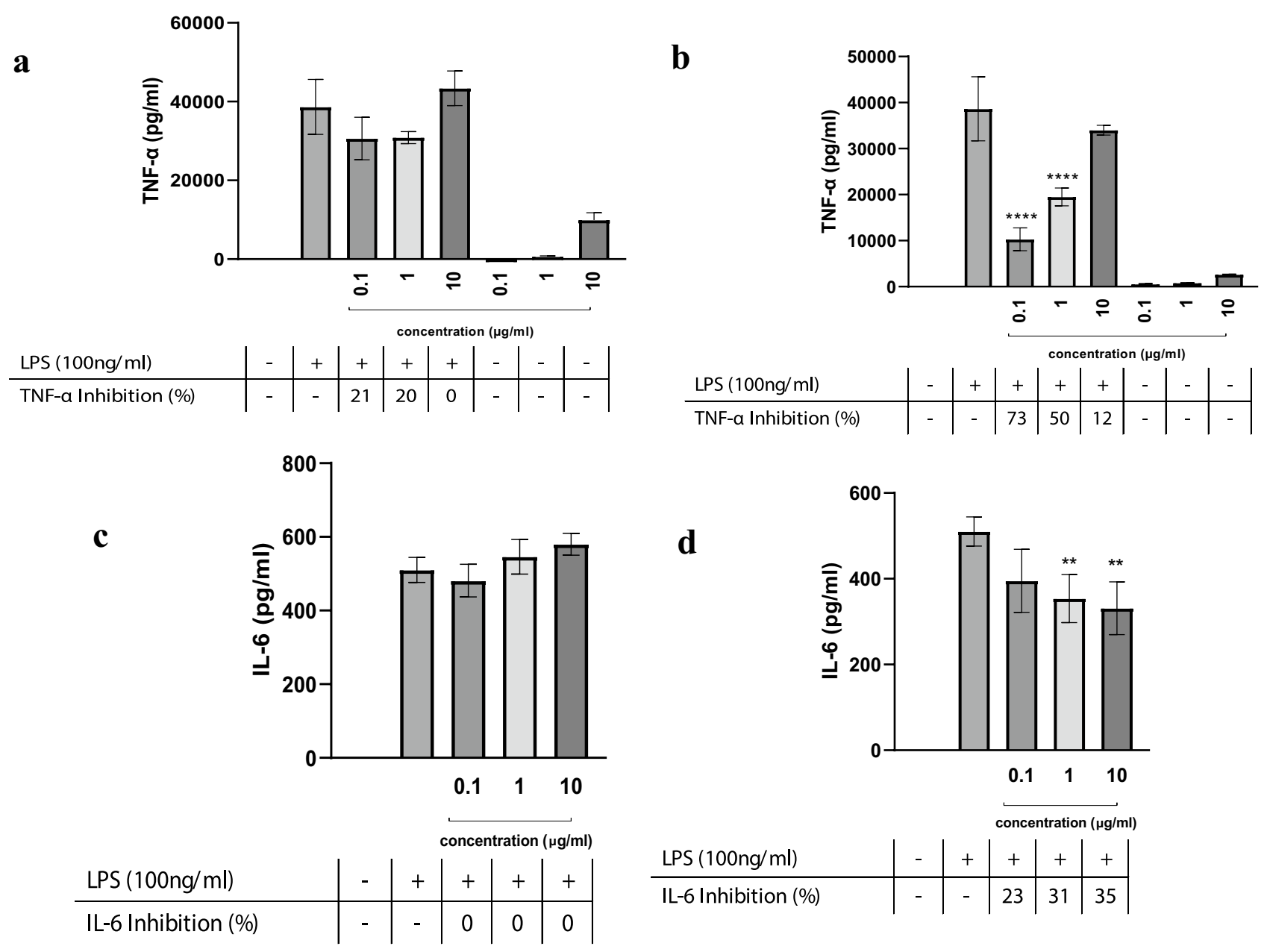

Fig. 2 The secretion of TNF- $\alpha$ or IL- 6 from LPS-induced RAW264.7 cells after treatment with various concentrations of Solar $(\mathbf{a}, \mathbf{c})$ and LED (b, d) Spirulina extracts. RAW264.7 were incubated with the indicated doses of spirulina extract in the presence or absence of LPS

for 4 h. TNF- $\alpha$ and IL- 6 secretion were determined by ELISA. Values are presented as means $\pm \mathrm{SD} ; * * p<0.01$, **** $p<0.001$ and compared with the LPS group

Lastly, in order to preserve a multi-batch consistent bioactivity of the extract, it is essential that the algal biomass is cultivated under consistent and controlled conditions throughout the year (e.g., light composition, irradiation level, temperature, $\mathrm{pH}$ levels). Such cultivation conditions may be realized in a fully controlled, indoor system, uninterrupted by diurnal or seasonal variations.

\section{Implications and Future Research}

We suggest at least three advantages of this novel approach of a natural, algae-based CS shield, and note that it requires further research.

First, as TNF- $\alpha$ is a key player in many acute inflammatory reactions, acting as an amplifier of inflammation, algaebased anti-TNF blockade has the potential to assist in treating other virus-induced CS, such as COVID-19, influenza, and autoimmune-related inflammatory diseases.

Second, and on condition that in vivo experiments establish the effectiveness of LED Spirulina in reducing CS, our proposed novel treatment could be dispensed extensively without putting the health of patients at risk, since Spirulina is FDA approved as a dietary supplement, and since its administration is non-intrusive, and can be orally absorbed.

Third, if animal and clinical trials confirm the efficacy of this anti-TNF therapy at the rates reported above, and the substance is available to the general population, a robust therapeutic intervention can be expected that is independent of vaccines, their requisites and potential ramifications, including skilled healthcare workers in clinics and possible side effects, especially in vulnerable populations with chronic diseases, which could only be detected in phase IV clinical trial years after administration. 


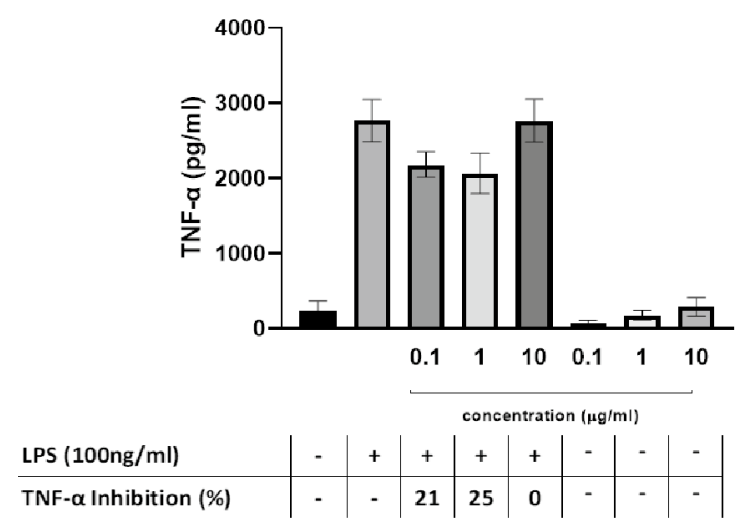

Fig. 3 The secretion of TNF- $\alpha$ from LPS-induced THP-1 human monocyte cells by the treatment of various concentrations of the Solar (a) or LED Spirulina (b) extracts. THP-1 cells were incubated with the indicated dose of Spirulina in the presence or absence of

Concomitantly, an algae-based anti-TNF therapy should be unaffected by variations in SARS-CoV-2 genome across major mutation clusters (Toyoshima et al. 2020), including recent mutations in SARS-CoV-2 that have raised concerns in regard to vaccine efficacy (Korber et al. 2020; Kupferschmidt 2020).

An ancillary, yet substantial benefit, such as therapeutic intervention-should it be proven efficient in human subjects-will free up capacity of ICUs, enable patients to receive over-the-counter affordable treatment option.

Drawing on our in vitro results, we stress the need for prompt in vivo studies to assess the effectiveness of various Spirulina extracts among animal models, as well as in clinical trials.

In addition, future research should note that the effect on TNF- $\alpha$ expression was greater than on IL-6. This finding suggests that the extract targets TNF- $\alpha$ secretion selectively and further validation with a more detailed mechanism of the anti-inflammation cascade within macrophages and monocyte cells should be explored.

It should likewise be noted that the extract from the LED-controlled cultivation process was significantly more effective at suppressing release of TNF- $\alpha$. To date, no natural compound has been proven to suppress specifically TNF- $\alpha$. Therefore, LED Spirulina could pave the way for novel algae-based bioactive compounds as anti-TNF treatment.

Acknowledgements Authors would like to thank Ms Kristina Atanasova for designing the graphical abstract and Mr. Tamir Avni for critical review of the manuscript.

Funding This EIT Food activity has received funding from the European Institute of Innovation and Technology (EIT), a body of the b

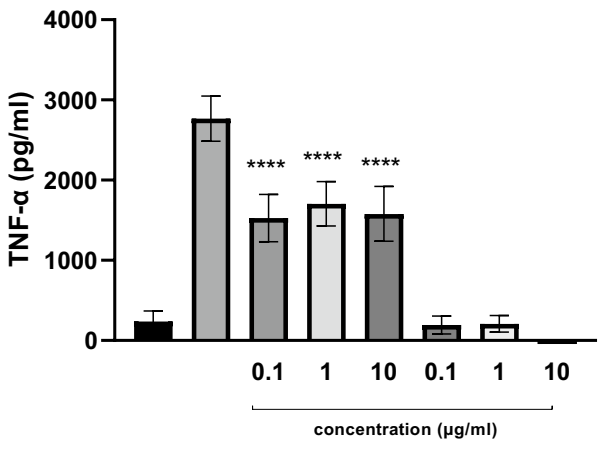

\begin{tabular}{l|c|c|c|c|c|c|c|c|} 
LPS (100ng/ml) & - & + & + & + & + & - & - & - \\
\hline TNF-a Inhibition (\%) & - & - & 42 & 38 & 44 & - & - & -
\end{tabular}

LPS for $2 \mathrm{~h}$. TNF- $\alpha$ secretion was determined by ELISA. Values are presented as means $\pm \mathrm{SD} ; * * * * p<0.001$ and compared with the LPS group

European Union, under Horizon2020, the EU Framework Program for Research and Innovation. The funding was received as part of the EIT's Crisis Response Initiative. This activity directly contributes to the European Union's response to the COVID-19 pandemic.

Data Availability Methods, materials, and data used in this study are fully delineated in the text.

\section{Compliance with Ethical Standards}

Conflict of Interest Authors declare no conflict of interests

Open Access This article is licensed under a Creative Commons Attribution 4.0 International License, which permits use, sharing, adaptation, distribution and reproduction in any medium or format, as long as you give appropriate credit to the original author(s) and the source, provide a link to the Creative Commons licence, and indicate if changes were made. The images or other third party material in this article are included in the article's Creative Commons licence, unless indicated otherwise in a credit line to the material. If material is not included in the article's Creative Commons licence and your intended use is not permitted by statutory regulation or exceeds the permitted use, you will need to obtain permission directly from the copyright holder. To view a copy of this licence, visit http://creativecommons.org/licenses/by/4.0/.

\section{References}

Alkuzweny M, Raj A, Mehta S (2020) Preparing for a COVID-19 surge: ICUs. E Clinical Medicine 25

Belay A, Ota Y, Miyakawa K, Shimamatsu H (1993) Current knowledge on potential health benefits of Spirulina. J Appl Phycol 5:235-241

Cherng SC, Cheng SN, Tarn A, Chou TC (2007) Anti-inflammatory activity of c-phycocyanin in lipopolysaccharide-stimulated RAW 264.7 macrophages. Life Sci 81:1431-1435

Chu WL, Lim YW, Radhakrishnan AK, Lim PE (2010) Protective effect of aqueous extract from Spirulina platensis against cell 
death induced by free radicals. BMC Compl Aternative medicine 10:53

Cian RE, López-Posadas R, Drago SR, Medina FSD, Martínez-Augustin $O$ (2012) Immunomodulatory properties of the protein fraction from Porphyra columbina. J Agric Food Chem 60:8146-8154

Coperchini F, Chiovato L, Croce L, Magri F, Rotondi M (2020) The cytokine storm in COVID-19: an overview of the involvement of the chemokine/chemokine-receptor system. Cytokine Growth Factor Rev

De Morais MG, Vaz BS, De Morais EG, Costa JAV (2015) Biologically active metabolites synthesized by microalgae. Biomed Res Int 2015, 15.

Feldmann M, Maini RN, Woody JN, Holgate ST, Winter G, Rowland M, Hussell T (2020) Trials of anti-tumour necrosis factor therapy for COVID-19 are urgently needed. The Lancet 395:1407-1409

Furmaniak MA, Misztak AE, Franczuk MD, Wilmotte A, Waleron M, Waleron KF (2017) Edible cyanobacterial genus Arthrospira: actual state of the art in cultivation methods, genetics, and application in medicine. Front Microbiol 8:2541

Hu B, Guo H, Zhou P, Shi ZL (2020) Characteristics of SARS-CoV-2 and COVID-19. Nat Rev Microbiol 1-14

Ishikawa T (2012) Clinical preparedness for cytokine storm induced by the highly pathogenic $\mathrm{H} 5 \mathrm{~N} 1$ influenza virus. J Pharmacogenomics Pharmacoproteomics 3:e131

Jafarzadeh A, Chauhan P, Saha B, Jafarzadeh S, Nemati M (2020). Contribution of monocytes and macrophages to the local tissue inflammation and cytokine storm in COVID-19: Lessons from SARS and MERS, and potential therapeutic interventions. Life Scie 118102

Jiang L, Wang Y, Yin Q, Liu G, Liu H, Huang Y, Li B (2017) Phycocyanin: a potential drug for cancer treatment. J Cancer 8:3416

Korber B, Fischer WM, Gnanakaran S, Yoon H, Theiler J, Abfalterer W, Montefiori DC (2020) Tracking changes in SARS-CoV-2 Spike: evidence that D614G increases infectivity of the COVID-19 virus. Cell 182:812-827

Kupferschmidt K (2020) Mutant coronavirus in the United Kingdom sets off alarms, but its importance remains unclear. Science December 20

McGonagle D, Sharif K, O'Regan A, Bridgewood C (2020) Interleukin-6 use in COVID-19 pneumonia related macrophage activation syndrome. Autoimmun Rev 102537

Mehta P, McAuley DF, Brown M, Sanchez E, Tattersall RS, Manson JJ, Across SpecialityCollaboration HLH (2020) COVID-19: consider cytokine storm syndromes and immunosuppression. Lancet (London, England) 395:1033

Minhas AK, Hodgson P, Barrow CJ, Adholeya A (2016) A review on the assessment of stress conditions for simultaneous production of microalgal lipids and carotenoids. Front Microbiol 7:546

Mittermaier M, Pickerodt P, Kurth F, de Jarcy LB, Uhrig A, Garcia C, Machleidt F, Pergantis P, Weber S, Li Y, Breitbart A, Bremer F, Knape P, Dewey M, Doellinger F, Weber-Carstens S, Slutsky AS, Kuebler WM, Suttorp N, Müller-Redetzky H (2020) Evaluation of PEEP and prone positioning in early COVID-19 ARDS. E Clin Med 28:100579
Moghadas SM, Shoukat A, Fitzpatrick MC, Wells CR, Sah P, Pandey A, Galvani AP (2020) Projecting hospital utilization during the COVID-19 outbreaks in the United States. Proc Natl Acad Sci 117:9122-9126

Mongkhon JM, Thach M, Shi Q, Fernandes JC, Fahmi H, Benderdour M (2014) Sorbitol-modified hyaluronic acid reduces oxidative stress, apoptosis and mediators of inflammation and catabolism in human osteoarthritic chondrocytes. Inflamm Res 63:691-701

Ooms MD, Dinh CT, Sargent EH, Sinton D (2016) Photon management for augmented photosynthesis. Nat Commun 7:1-13

Otegbade OO, Ojo JA, Adefokun DI, Abiodun OO, Thomas BN, Ojurongbe O (2017) Ethanol extract of Blighia sapida stem bark show remarkable prophylactic activity in experimental Plasmodium berghei-infected mice. Drug Target Insights 11:1177392817728725

Rajasekaran C, Ajeesh CM, Balaji S, Shalini M, Ramamoorthy SIVA, Ranjan DAS, Kalaivani T (2016) Effect of modified Zarrouk's medium on growth of different Spirulina strains. Walailak J Scie Technol (WJST) 13:67-75

Robinson PC, Richards D, Tanner HL, Feldmann M (2020) Accumulating evidence suggests anti-TNF therapy needs to be given trial priority in COVID-19 treatment. Lancet Rheumatol 2:e653-e655

Ruan Q, Yang K, Wang W, Jiang L, Song J (2020) Clinical predictors of mortality due to COVID-19 based on an analysis of data of 150 patients from Wuhan. China Intensive Care Medicine 46:846-848

Saini MK, Sanyal SN (2015) Cell cycle regulation and apoptotic cell death in experimental colon carcinogenesis: intervening with cyclooxygenase-2 inhibitors. Nutr Cancer 67:620-636

Shimizu M (2019) Clinical Features of Cytokine Storm Syndrome. In Cytokine Storm Syndrome (pp. 31-41). Springer, Cham

Toyoshima Y, Nemoto K, Matsumoto S, Nakamura Y, Kiyotani K (2020) SARS-CoV-2 genomic variations associated with mortality rate of COVID-19. J Hum Genet 65:1075-1082

Vandenberg LN (2014). Non-monotonic dose responses in studies of endocrine disrupting chemicals: bisphenol a as a case study. Doseresponse 12

Ye Q, Wang B, Mao J (2020) The pathogenesis and treatment of the Cytokine Storm' in COVID-19. J Infect 80:607-613

Publisher's Note Springer Nature remains neutral with regard to jurisdictional claims in published maps and institutional affiliations. 